\title{
Kernos
}

Revue internationale et pluridisciplinaire de religion grecque antique

12 | 1999

Varia

\section{Le péan d'Isyllos : forme et finalités d'un chant religieux dans le culte d'Asklépios à Épidaure}

\section{Pierre Sineux}

\section{(2) OpenEdition \\ Journals}

Édition électronique

URL : http://journals.openedition.org/kernos/717

DOI : $10.4000 /$ kernos.717

ISSN : 2034-7871

Éditeur

Centre international d'étude de la religion grecque antique

Édition imprimée

Date de publication : 1 janvier 1999

Pagination : 153-166

ISSN : 0776-3824

Référence électronique

Pierre Sineux, «Le péan d'Isyllos : forme et finalités d'un chant religieux dans le culte d'Asklépios à Épidaure », Kernos [En ligne], 12| 1999, mis en ligne le 13 avril 2011, consulté le 01 mai 2019. URL: http://journals.openedition.org/kernos/717 ; DOI : 10.4000/kernos.717 


\section{Le péan d'Isyllos : forme et finalités d'un chant religieux dans le culte d'Asklépios à Épidaure}

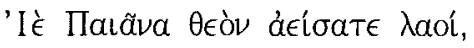

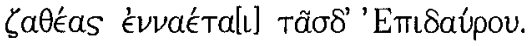

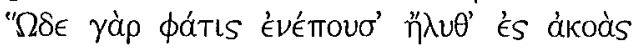

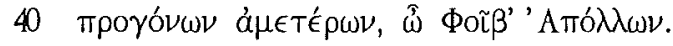

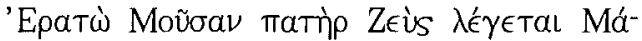

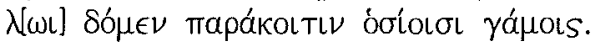

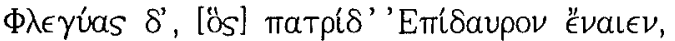

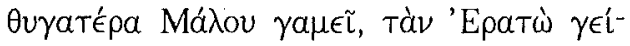

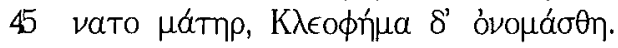

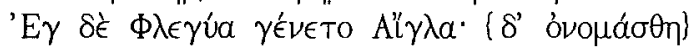

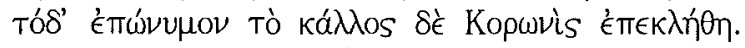

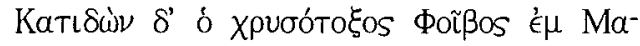

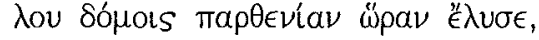

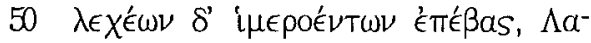

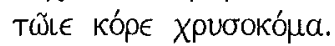

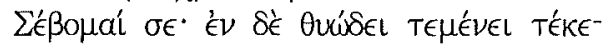

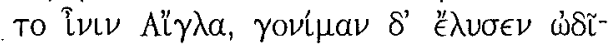

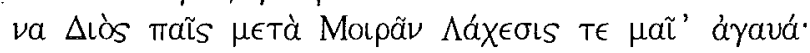

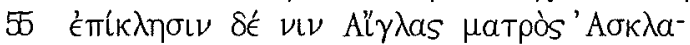

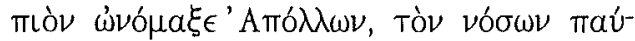

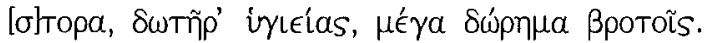

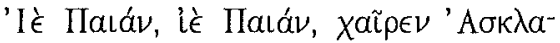

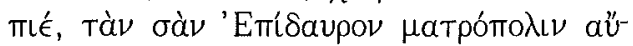

$60 \xi \omega \nu, \dot{\epsilon} \nu \alpha \rho \gamma \eta \tilde{~} \delta$ '

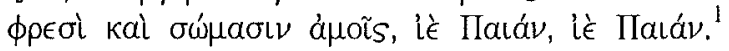

Ié, chantez, vous tous, le dieu Paian,

habitants de cette vénérable Épidaure.

Car voici ce que dit l'histoire qui vint aux oreilles

de nos ancêtres, ô Phoibos Apollon.

On dit que Zeus le père donna la Muse Ératô

comme épouse à Malos en justes noces.

1 Nous suivons l'édition de L. KÄppel, Paian. Studien zur Gescbichte einer Gattung, Berlin-New York, 1992, p. 380-383, Pai 40 (E; 1. 37-61) (avec apparat critique); le dialecte est, sauf exceptions, dorien; le mètre est ionique. 
Et Phlégyas, qui habitait Épidaure, sa patrie, épousa la fille de Malos, que sa mère Ératô avait mise au monde; son nom était Kléophéma.

De Phlégyas, une enfant naquit, son nom était Aigla; c'était son nom mais à cause de sa beauté, elle était aussi appelée Coronis.

Et Apollon aux flèches d'or, de là-haut l'ayant remarquée dans la maison de Malos, mit fin au temps de sa virginité.

Sur sa couche désirable, tu montas, fils de Létô aux cheveux d'or!

Je te révère; dans le sanctuaire au parfum d'encens, Aigla mit au monde un enfant, et des douleurs de l'enfantement la délivrèrent le fils de Zeus avec les Moires et Lachésis, l'admirable sage-femme.

Suivant le nom de sa mère Aigla, Apollon le nomma Asklapios, celui qui fait cesser les maladies, le dispensateur de la santé, un grand bienfait pour les mortels. Ié Paian, ié Paian, salut Asklapios, fais croître la cité de ta mère, Épidaure, envoie une santé éclatante à nos esprits et à nos corps, ié Paian, ié Paian.

Le péan d'Isyllos, que nous reproduisons ici, est un élément d'une inscription de soixante-dix neuf lignes découverte à la fin du siècle dernier, à l'est du temple d'Asklépios dans le sanctuaire d'Épidaure ${ }^{2}$. L'inscription dans sa totalité a fait l'objet de plusieurs éditions et sa lecture ne pose guère de problèmes ${ }^{3}$. La datation la plus couramment proposée oscille entre 300 et 280 av. J.-C. ${ }^{4}$ : l'inscription est donc contemporaine de la fin des grands pro-

2 P. Kavvadias, Fouilles d'Épidaure, I, Athènes, 1893, p. 34-36; stèle en pierre calcaire. Haut. : $127 \mathrm{~cm}$; larg. : $53 \mathrm{~cm}$; ép. : $10 \mathrm{~cm}$. Inscription stoichèdon, actuellement au musée d'Épidaure.

3 P. Kavvadias, ArcbEph (1885), p. 65 sq.; F. Blass, Jabrbücber für class. Pbilol., 131 (1885), p. 822-826; U. von Wilamowitz Moellendorf, Isyllos von Epidauros, Berlin, 1886 (Philologische Untersuchungen, IX), p. 13 sq.; H. Collitz - F. BECHTEL, SGDI, III, 1, 3342;

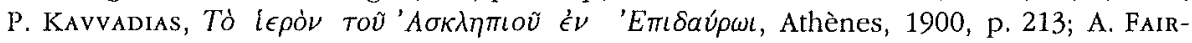
BANKs, A Study of the Greek Paean, New York, 1900 (Cornell Studies in Classical Pbilology, XII), p. 109-110; J.U. Powell, Collectanea Alexandrina, Oxford, 1925, p. 132-136; F. Himler von Gaertringen, IG IV ${ }^{2}, 1,128$ (M. Fraenkel, $I G$ IV, 950); E.J. et L. Edelstein, Asclepius. $A$ collection and interpretation of the testimonies, I, Baltimore, 1945, T. 18, 32, 295, 296, 516 , 594; la numérotation des lignes utilisée dans la suite de notre texte renvoie à l'édition de L. KäPPEL, op. cit. (n. 1), Pai 40.

4 D'une part, la forme des lettres est la même que sur les deux premières « stèles de guérisons " (deuxième moitié du $\mathrm{Iv}^{\mathrm{e}}$ siècle) à l'exception du $\nu$ et du $\pi$ qui indiqueraient que l'inscription d'Isyllos est quelque peu postérieure : $c f$. P. Kavvadias, Fouilles d'Épidaure, p. 34; U. von Wilamowitz, op. cit. (n. 3), p. 34. D'autre part, la fin de l'inscription contient le récit d'un miracle auquel le poète, alors qu'il était un enfant, a assisté. Le dieu Asklépios lui serait apparu «en ces temps où Philippe conduisait son armée contre 
grammes d'aménagement du sanctuaire d'Asklépios qui a vu notamment se succéder les constructions du temple, du portique d'incubation (à partir de 370 av. J.-C. environ), de la tholos et du théâtre ${ }^{5}$.

L'inscription dans son ensemble se présente sous la forme d'une dédicace d'Isyllos - un poète d'Épidaure, si l'on en juge d'après l'inscription elle-même - à Apollon Maléatas et à Asklépios (1. 1-2) et elle comprend six paragraphes plus ou moins explicitement reliés entre eux. Le premier (1. 3-9) fait l'éloge de l'aristocratie; le second mentionne une loi sacrée qu'Isyllos a lui-même proposée et qui a été votée par la cité d'Épidaure (1. 10-26); cette loi réglemente une procession en l'honneur d'Apollon et d'Asklépios pour laquelle on choisira par tribu, ceux qui « seront les meilleurs »; ces derniers conduiront la procession et prieront les divinités pour qu'elles apportent la bonne santé aux citoyens d'Épidaure et garantissent leur kalokagatbia, ainsi que l'eunomia, la paix et la richesse dans la cité. Le paragraphe suivant (1. 27-31) attribue la fondation du sanctuaire d'Apollon Maléatas à Malos, présenté plus loin comme ancêtre d'Asklépios. Ensuite, une sorte de préambule au péan proprement dit rapporte qu'à la demande du poète, l'oracle de Delphes a fait savoir qu'il serait préférable de faire graver le péan sur la pierre (1. 32-36). Enfin, après le péan (1. 37-61), Isyllos fait le récit d'un « miracle » d'Asklépios (1. 62-84) : alors qu'Isyllos était lui-même enfant et malade, le dieu lui était apparu et lui avait annoncé qu'il le soignerait; mais, au préalable, le dieu devait se rendre à Sparte pour sauver la cité attaquée par Philippe. Isyllos était alors allé annoncer la nouvelle à Sparte qu'Asklépios sauva.

À l'intérieur de cet ensemble, il s'agit de voir comment Isyllos, tout en respectant les règles formelles voulues par la « loi du genre » du péan, dote ce dernier d'un certain nombre de finalités qui dépassent celles que l'on attend d'une prière chantée: Isyllos défend, en effet, une conception singulière de l'histoire du sanctuaire d'Épidaure et de la divinité Asklépios et met sa prière à Asklépios au service d'un projet de nature finalement politique.

Dans son étude sur les hymnes, J.M. Bremer a repris et discuté l'ancienne classification tripartite établie par K. Ausfeld (invocatio, pars epica, preca-

\footnotetext{
Sparte » : la plupart des auteurs s'accordent à voir là une allusion aux opérations menées par Philippe II de Macédoine dans, le Péloponnèse et, en particulier, l'invasion, par ce dernier, de la vallée de l'Eurotas, après la bataille de Chéronée. Or, l'inscription a été écrite au temps de la maturité d'Isyllos puisqu'il évoque ses opinions politiques au début de l'inscription en affirmant n'en avoir pas changé entre « jadis » et « maintenant ». Cette inscription peut donc provenir des premières années du II $^{\mathrm{e}}$ siècle. F. BLAss, art. cit. (n. 3), prétend toutefois que le roi mentionné est Philippe $\mathrm{V}$ qui mena une expédition dans le Péloponnèse en 218; hypothèse réfutée avec de bons arguments par R. Herzog, Die Wunderbeilungen von Epidauros, Leipzig, 1931 (Pbilologus, Supplement, 22, 3), p. 41-42. Voir aussi P. Cartledge, A. Spawforth, Hellenistic and Roman Sparta: A Tale of Two Cities, Londres-New York, 1989, p. 14.

5 Cf. G. Roux, L'architecture de l'Argolide aux IVe et III siècles av. J.-C., Paris, 1961; R.A. Tomlinson, Epidauros, Londres, 1983 (BEFAR 199).
} 
tio) ${ }^{6}$. S'accordant sur l'essentiel avec K. Ausfeld, J.M. Bremer propose cependant, à juste titre, le terme d'« argument » pour désigner la partie intermédiaire qui ne comprend de développements selon les modes de la poésie épique que dans certains Hymnes bomériques ${ }^{7}$. De surcroît, cet « argument » sert avant tout de fondement à la demande qui termine la prière sur le mode «puisque tu as tel et tel pouvoir, accède à ma demande qui est... ${ }^{8}$.

Le péan d'Isyllos obéit apparemment à cette structure tripartite. Le péan

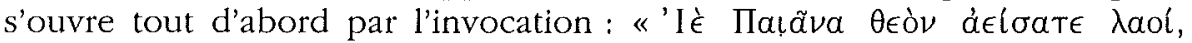

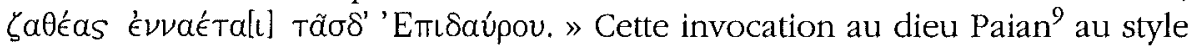
indirect renseigne sur les conditions de la performance et ses acteurs : l'ex-

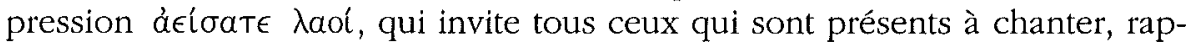

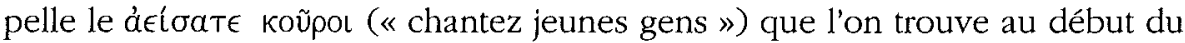
péan d'Érythrées en l'honneur d'Asklépios ${ }^{10}$. Dans les deux cas, nous sommes bien en présence d'un hymne cultuel mais, alors que le chant d'Érythrées est entonné par un chœur de jeunes gens, le péan d'Isyllos est chanté par un plus grand nombre : il s'agit soit d'un péan processionnel - chanté peut-être lors de la procession instituée par la loi sacrée qui avait été proposée par Isyllos lui-même ${ }^{11}$-, soit d'un péan entonné par les participants à un rituel public, par exemple un sacrifice ${ }^{12}$.

6 K. AUSFELD, De Graecorum precationibus quaestiones, in Jabrbücber für class. Phil., 28 (1903), p. 505 sq.; J.M. Bremer, Greek Hymns, in H.S. Versnel, Faith, Hope and Worship, Leiden, 1981, p. 193-215. Cf. également W.D. Furlex, Praise and Persuasion in Greek Hymns, in JHS 115 (1995), p. 29-46, en particulier p. 35.

7 C. Calame, Variations énonciatives, relations avec les dieux et fonctions poétiques dans les Hymnes Homériques, in $M H, 52$ (1995), p. 15, constatant que cette partie est spécifiquement consacrée à l'éloge du dieu, propose d'ailleurs que l'on parle d'« epica laus » plutôt que d'« epica pars" .

8 On peut trouver ici le rappel d'une circonstance dans laquelle le dieu s'est déjà manifesté; d'où l'appellation de bypomnesis que retient, pour la seconde partie de l'hymne cultuel, A.M. Milder, From Delos to Delphi. A literary study of the Homeric Hymn to Apollo, Leiden, 1986, p. 1-9.

9 Sur le sens de Paian, cf. infra.

10 F. Sokolowski, Lois sacrées de l'asie Mineure, Paris, 1955, $n^{\circ} 24 ;$ H. Engelmann R. Merkelbach, Die Inschriften von Erythrai und Klazomenai, 1973, $n^{\circ}$ 205; L. KäPPEL, op. cit. (n. 1), Pai. 37 et p. 189-206. Le péan d'Érythrées figure au verso d'une stèle gravée dans les années 380-360 av. J.-C., au moment vraisemblablement où fut introduit le culte d'Asklépios à Érythrées; le recto contient une loi sacrée se rapportant au rite de l'incubation; le verso contient un péan à Apollon, très mutilé, et un péan à Asklépios. On connaît trois autres rédactions du péan à Asklépios, en provenance d'Athènes, de Ptolémaïs en Égypte et de Dion en Macédoine : cf. F. Sokolowski, op. cit., p. 65.

11 U. von WILAMOWITZ, op. cit. (n. 3), p. 44 sq.

12 Sur la "performance » des hymnes : J.M. Bremer, art. cit. (n. 6), p. 197 sq. Nous connaissons de nombreux exemples d'hymnes et de péans chantés au début ou après un sacrifice : les jeunes filles devaient chanter un péan au début du sacrifice à Artémis dans Eur., Iph. Aul., 1467-1484; l'Hymne Crétois au Kouros est un hymne chanté à ou autour

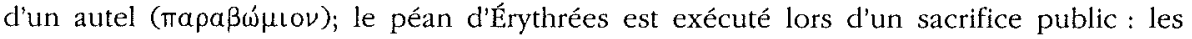


La partie intermédiaire du péan d'Isyllos présente ensuite une généalogie développée du dieu suivie d'une évocation des circonstances de sa naissance (1. 36-56); le passage prend la forme d'un long récit (entrecoupé de deux invocations à Apollon sur lesquelles nous reviendrons) dont Isyllos prend soin de préciser qu'il n'en est pas l'auteur mais qu'il s'agit d'une histoire qu'il

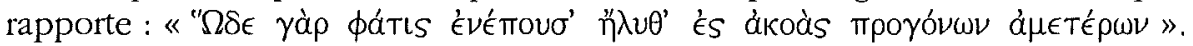

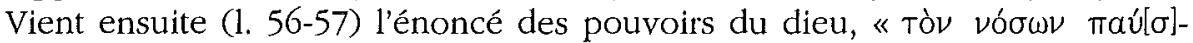

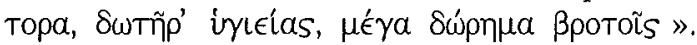

Cet énoncé des pouvoirs du dieu est attendu dans la mesure où il fonde la demande formulée dans la troisième partie. Le développement sur la généalogie, quant à lui, n'est pas sans rappeler, à première vue, le péan d'Érythrées dont la partie intermédiaire contient une assez longue présentation de la famille d'Asklépios : «Le fils de Létô qui frappe au loin, iè Paian, lui qui a engendré une grande joie pour les mortels, quand il s'unit en amour avec Coronis dans le pays de Phlégyas, iè Paian, Asklépios, daimôn des plus illustres, iè Paian. Et de lui sont nés Machaon et Podaleirios, et Iaso, iè Paian, et Aigla la jolie et Panakeia, enfants d'Épionê, ainsi que la glorieuse et resplendissante Hygieia, iè Paian, Asklépios, daimôn des plus illustres, iè Paian $^{13}$. " Dans ce passage, l'évocation de la naissance est limitée au fait

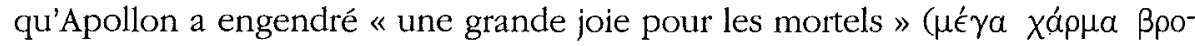
Toĩ $(\nu)$, expression traditionnelle que l'on retrouve sous la forme " grand

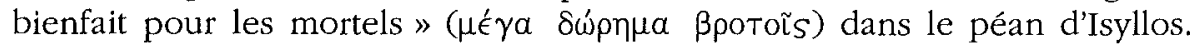
Toutefois, la comparaison s'arrête là et, si la désignation des enfants d'Asklépios pourrait rappeler la présentation de la généalogie dans le péan d'Isyllos, la signification de l'un et l'autre passages s'avère très différente. Dans le péan d'Érythrées, en effet, la désignation des enfants d'Asklépios sert à fonder la demande de santé : leurs noms évoquent tous, à l'exception de Machaon et Podaleirios qui sont cependant bien connus comme héros guérisseurs, différents aspects de la santé. Iaso est la Guérison, Aigla est la Rayonnante,

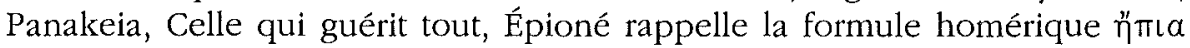
$\phi a ́ p \mu a k a$ (les remèdes apaisants) et Hygieia constitue une personnification de la Santé ${ }^{14}$. Ainsi, ce sont les différents effets des pouvoirs du dieu qui se trouvent ainsi énumérés et cette énumération, par le biais de la descendance du dieu, constitue « l'argument » de la prière. Par comparaison, la généalogie présentée par Isyllos apparaît comme détachée de la structure d'ensemble, nous y reviendrons.

La pétition, enfin, constitue la demande adressée à la divinité. Elle s'ouvre

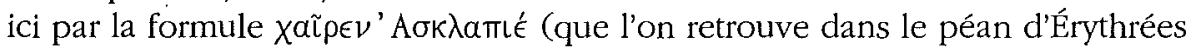

participants pouvaient sans doute, dans la mesure de leurs possibilités, se joindre à l'exécution du chant ou, au moins, aux exclamations; $c f$. D. Aubriot-Sevin,. Prière et conceptions religieuses en Grèce ancienne jusqu'à la fin du ve siècle av.J.-C., Lyon, 1992, p. $176 \mathrm{sq}$.

13 Nous suivons l'édition de L. KäppeL, op. cit. (n. 1), Pai 37.

14 J.M. BremEr, art. cit. (n. 6), p. 209; L. KÄPPEL, op. cit. (n. 1), p. 194-195. 
sous la forme $\chi a i n \epsilon \mu o l)$. Le terme est plus qu'un simple salut, destiné qu'il est à attirer la bienveillance du dieu. Il met en évidence la relation que celui qui accomplit le chant (ici la communauté) essaie d'établir avec le dieu : les gens espèrent que le chant qui est destiné à le flatter et à le charmer réjouira le dieu. Le but est d'attirer son attention pour recevoir, en retour, une grâce ou une faveur, c'est-à-dire en définitive une réjouissance. La relation de $\chi \alpha$ á $\rho$ s est ainsi une relation qui implique une réciprocité et cette notion est essentielle dans tous les hymnes grecs ${ }^{15}$. De façon logique, le terme apparaît ici immédiatement avant la requête. La forme de cette dernière découle des pouvoirs du dieu tels qu'ils ont été établis dans le corps de l'hymne et procède en deux temps comme on peut s'y attendre ${ }^{16}$ : le premier bénéficiaire en est la cité tout entière ( « fais croître la cité de ta mère, Épidaure »), puis ceux au nom de qui le chant est exécuté ( $«$ envoie une santé éclatante à nos esprits et à nos corps $»)^{17}$.

Ainsi, l'examen de la structure du péan d'Isyllos révèle bien sa nature de "prière chantée »; chacune des parties remplit une fonction précise et l'ensemble prépare et aboutit à la demande de santé pour ceux qui prononcent le chant. L'auteur dit lui-même qu'il s'agit d'un péan ${ }^{18}$. Or, un péan est à proprement parler un hymne destiné au culte d'Apollon ${ }^{19}$ et s'il a pu parfois être adressé à d'autres divinités (Artémis, Dionysos...), il n'a pas, au moins jusqu'au Iv ${ }^{e}$ siècle, perdu cette connexion originelle avec Apollon ${ }^{20}$. Les péans adressés à d'autres divinités qu'Apollon prennent bien soin de souligner les liens que ces divinités entretiennent avec ce dernier ${ }^{21}$. Isyllos ne déroge pas à

15 Sur cette notion, $c f$. W.H. RACE, Aspects of Rbetoric and Form in Greek Hymns, in GRBS, 23 (1982), p. 5-14; W.D. FURLEY, art.cit. (n. 6), p. 32; C. CALAME, art.cit. (n. 7), p. 10-11.

16 W.H. RACE, art. cit. (n. 15), p. 10-14. Il est clair que le long développement de la généalogie du dieu, s'il peut se justifier éventuellement par une volonté de plaire au dieu, ne fonde pas, d'une manière stricte, la requête qui est formulée à la fin de la prière.

17 Le péan d'Érythrées présente, dans sa troisième partie, ces deux aspects et aboutit également à une demande de santé : «viens dans ma cité spacieuse pour lui être propice » puis « donne-nous la joie de voir la lumière agréable du soleil ainsi que la glorieuse et resplendissante Hygieia ».

18 C'est ainsi qu'Isyllos désigne son chant : «... au sujet du péan qu'il avait composé pour Apollon et pour Asklapios, s'il était mieux pour lui de faire graver le péan sur la pierre » (1. 33-35).

19 Certains auteurs ont cherché à établir une distinction radicale entre " hymne " et "péan » en se fondant notamment sur Platon, Lois III, 700b et sur la classification de la poésie de Pindare par les Alexandrins qui séparent un livre d'hymnes des livres de péans, dithyrambes, hyporchèmes... : A.E. HARver, The Classification of Greek Lyric Poetry, in CQ n.s. 5 (1955), p. 157-175; L. KÄPPEL, op. cit. (n. 1), p. 64. Ce débat est clarifié dans W.D. Furley, art. cit. (n. 6), p. 31-32 qui montre comment une fausse distinction a pu émerger entre hymnes et autres poèmes lyriques de nature religieuse.

20 A. Fairbanks, op. cit. (n. 3), p. 66-67 en particulier; C.M. Bowra, Greek Lyric Poetry, Oxford, 1961, p. 408.

21 A. Fairbanks, op. cit. (n. 3), p. 48-49. 
cette règle et dit lui-même qu'il a composé un péan «pour Apollon et pour Asklépios » et associe de façon explicite les deux divinités dans un but qu'il nous faut examiner plus précisément.

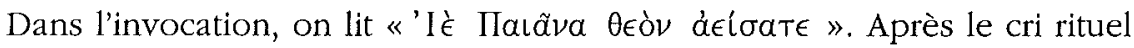
« lé », « Пaıãva $\theta \epsilon o ̀ \nu 》$ forme un ensemble à l'accusatif, complément d'objet du verbe chanter. Qui est ce « dieu Paian »? On sait que Paian est d'abord le nom d'un dieu médecin; mais Paian est aussi épithète d'Apollon ou le nom même de ce dieu avant d'être utilisé pour désigner Asklépios ${ }^{22}$. Ici, l'auditeur est renseigné au quatrième vers du péan où apparaît un vocatif sous la forme

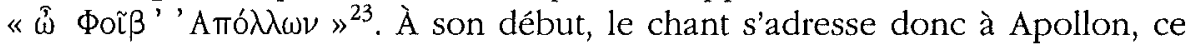
que confirme la rupture énonciative dans le récit de la généalogie : « $\lambda \epsilon \chi \epsilon \hat{\epsilon} \omega \nu$

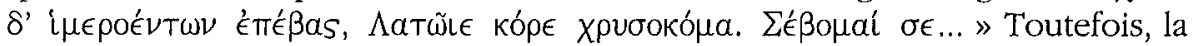

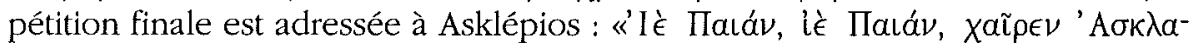

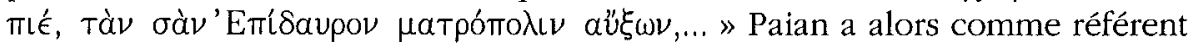
Asklépios. L. Käppel a montré qu'un glissement de même nature était à l'œuvre dans le péan d'Érythrées ${ }^{24}$ où, dans la première strophe, Paian désigne tout d'abord Apollon : «Chantez, jeunes gens, Paian célèbre pour sa sagesse, le fils de Létô qui frappe au loin, iè Paian, lui qui a engendré une grande joie pour les mortels, quand il s'unit en amour avec Coronis dans le pays de Phlégyas, iè Paian, Asklépios, daimôn des plus illustres, iè Paian. » C'est encore à Apollon que s'adresse le premier meshymnion car, à ce moment-là, il n'a pas encore été question d'Asklépios. Ensuite, à partir du refrain " iè Paian, Asklépios, daimôn des plus illustres, iè Paian », Paian désigne Asklépios; de même, c'est Asklépios qui est invoqué dans la demande du chœur à la fin du péan: "Réjouis-toi, viens dans ma cité spacieuse pour lui être propice, iè Paian, et donne-nous la joie..., iè Paian, Asklépios, daimôn des plus illustres, iè Paian. »

Dans l'un et l'autre poèmes, l'auditeur assiste donc au remplacement d'Apollon par Asklépios dans l'invocation à Paian. Ce changement de référent qui souligne le maintien du lien originel d'Apollon avec le péan est évidemment facilité par les liens généalogiques très étroits qui existent entre les deux divinités. Cependant, ce glissement a des implications importantes en ce qui concerne la relation qui s'établit, par le chant, entre la communauté et ces deux divinités. Il aboutit tout d'abord à donner au fils une position prééminente par rapport au père, puisque c'est par lui que s'achève la prière et que c'est à lui que s'adresse la requête finale. Surtout, il indique la spécificité

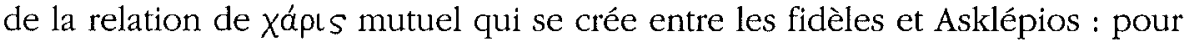
réjouir Asklépios, il faut aussi chanter Apollon et, pour obtenir une faveur du fils, il faut aussi et préalablement honorer le père. De ce point de vue, le péan traduit très exactement la nature des relations qui existent à Épidaure, dans

\footnotetext{
22 A. Fairbanks, op. cit. (n. 3), p. 4-13.

23 D'autres vocatifs d'Apollon sous la forme 'Amó $\lambda \lambda \omega \nu$ sont attestés (voir, par ex., Esch., Choéph., 559).

24 L. KAPPEL, op. cit. (n. 1), p. 194-196.
} 
les pratiques cultuelles, entre les deux divinités. C'est ainsi qu'au troisième paragraphe de son inscription, Isyllos rappelle cette nécessaire subordination : "Pas même à Tricca en Thessalie, tu ne saurais être tenté de descendre dans l'adyton d'Asklépios, sans avoir d'abord offert un sacrifice sur le pur autel d'Apollon Maléatas ${ }^{25}$. " «Descendre dans l'adyton d'Asklépios » est une expression qui désigne l'accomplissement du rite de l'incubation ${ }^{26}$; parmi les rites préliminaires à ce rite de l'incubation, il y avait donc un sacrifice à Apollon Maléatas et la tournure oủsé sous-entend qu'à Épidaure, tel était le cas. Un règlement cultuel du $\mathrm{rv}^{\mathrm{e}}$ siècle, retrouvé dans le sanctuaire d'Apollon Maléatas, situé sur le mont Kynortion, sur les hauteurs qui dominent le sanctuaire d'Asklépios, précise ce que le prêtre d'Asklépios d'une part et le prêtre d'Apollon d'autre part devront fournir à ceux qui accomplissent le sacrifice préliminaire à l'incubation ${ }^{27}$. Cette association cultuelle entre les deux divinités se retrouve à Épidaure selon bien d'autres modalités ${ }^{28}$ (mais aussi à Érythrées et dans un certain nombre d'autres sanctuaires d'Asklépios) $)^{29}$.

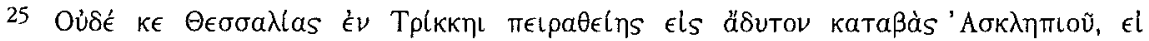

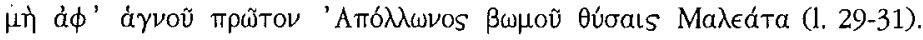

26 Le terme adyton est utilisé à Épidaure comme terme équivalent à celui d'abaton dans un des récits des "stèles de guérison »: R. Herzog, op. cit. (n. 4), W 38 (stèle B, 1. 111-112).

27 F. Sokolowski, Lois sacrées des cités grecques. Supplément, Paris, 1962, $\mathrm{n}^{\circ} 22$ et SEG, XXII, 276; A. Petropovlou, Prothysis and altar: a case study, in R. Étienne-M.-Th. Le Dinahet (éds), L'espace sacrificiel dans les civilisations méditerranéennes de l'Antiquité. Actes du colloque tenu à la Maison de l'Orient, Lyon, 4-7 juin 1988, Paris, 1991, p. 25-31.

28 Ainsi, par exemple, les «stèles de guérison » portent le titre « $\AA$ la Bonne Fortune. Guérisons d'Apollon et d'Asklépios »: R. Herzog, op. cit. (n. 4), (stèle A, 1. 1-2). Le fait qu'Apollon n'apparaisse pas dans les récits qui suivent n'empêche pas qu'on ait voulu l'associer aux guérisons. En outre, le sanctuaire d'Asklépios installé en contre-bas du sanctuaire d'Apollon Maléatas a été relié à ce dernier par une canalisation de sorte que l'eau utilisée dans les rites précédant le rite d'incubation provenait de ce sanctuaire d'Apollon Maléatas: S.G. Cole, The Uses of Water in Greek Sanctuaries, in R. HäGG, N. Marinatos, G. C. Nordquist (éds), Early Greek Cult Practice, Stockholm, 1988, p. 163; V. Lambrinoudaxis, Staatkult und Geschichte der Stadt Epidauros, in Archaiognosia 1 (1980), p. 56-57; R. Ginouvès, Balaneutiké, Recherches sur le bain dans l'Antiquité grecque, Paris, 1962 (BEFAR 200), p. 352.

29 Ainsi, à Pergame, d'après une loi sacrée (gravée vraisemblablement au ${ }_{\mathrm{II}}^{\mathrm{e}}$ ap. J.-C.), un double sacrifice à Apollon et à Asklépios devait être accompli avant l'incubation : M. Wörrle, Ch. Habicht, Altertümer von Pergamon, VIII, 3, Berlin, 1969, p. 168-169 et p. 175. Apollon fait partic des divinités qui reçoivent des popana comme offrandes préliminaires au sacrifice annuel à Asklépios au Pirée : $I G \mathrm{II}^{2}$, 4962; F. Sokolowski, Lois sacrées des cités grecques, Paris, 1969, $\mathrm{n}^{\circ} 21$. À Érythrées, dans le règlement relatif au culte d'Asklépios (380-360 av. J.-C.) déjà cité, des sacrifices doivent être faits à Apollon et à Asklépios après l'incubation: supra, n. 10 et F. Graf, Nordionische Kulte, Vevey, 1985, p. $250-257$. 
Dès lors, on voit comment la structure même du chant est déterminée par le souci de mettre en évidence et de maintenir, non seulement le lien originel entre Apollon et le péan, mais aussi l'ordre même du rituel du culte d'Asklépios : dans la prière, comme dans le rite de l'incubation, pour accéder au fils, il faut en passer par le père. Apollon, prédécesseur d'Asklépios en tant que dieu guérisseur, reste présent au début du péan adressé à Asklépios, de la même façon qu'il reste présent à l'intérieur des rites asclépieiens.

Il n'en reste pas moins que l'ampleur remarquable du récit de la généalogie fait du péan d'Isyllos en tant qu'hymne cultuel un cas limite. Cette généalogie se déroule sur cinq générations, étagées de la façon suivante :

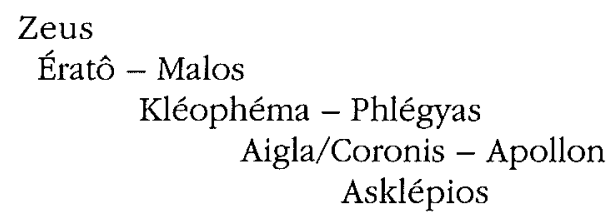

Cette généalogie, on le voit, s'écarte assez largement de toutes les traditions connues se rapportant à Asklépios et semble avoir pour but premier d'établir la primauté du sanctuaire d'Épidaure sur le sanctuaire de Tricca en Thessalie (le plus ancien sanctuaire d'Asklépios selon une tradition courante, transmise notamment par Strabon ${ }^{30}$ ). L'un des personnages-clés de cette organisation est Malos que l'on ne rencontre dans aucune tradition concernant Asklépios. Le troisième paragraphe de l'inscription d'Isyllos éclaire cette intégration de Malos à la généalogie : « le premier, Malos, a construit un autel d'Apollon Maléatas et par des sacrifices a magnifié son domaine ${ }^{31}$. » Malos, ancêtre d'Asklépios, figure ici comme le fondateur du premier sanctuaire d'Apollon Maléatas et le premier à avoir effectué un sacrifice sur son autel. Les Épidauriens qui exécutent ou écoutent le chant ne peuvent manquer de reconnaître dans cette mention le vieux sanctuaire apollinien du Mont Kynortion tout proche et étroitement relié au sanctuaire d'Asklépios. Le nom même de Malos semble venir justifier l'épiclèse Maléatas ${ }^{32}$. Dès lors, tous les autels d'Apollon Maléatas présents ici ou là sont postérieurs à celui d'Épi-

30 Strabon, IX, 5, 17. De son côté, Hérondas, Mim., II, 97, affirme que c'est de Tricca qu'Asklépios serait venu à Cos. En outre, les Épidauriens avaient affaire à toute la tradition véhiculée par l'épopée homérique selon laquelle Asklépios était originaire de Tricca et père de deux guerriers, Machaon, «l'irréprochable médecin » et Podaleirios : $I l$, II, 729-733; IV, 193-219; XI, 833-837: Les fouilles archéologiques effectuées à Tricca n'ont pu confirmer l'ancienneté du sanctuaire de Tricca; $c f$. J. ZieneN, MDAI(A), 17 (1892), p. 195197; Kastriotis, ArchEph, 35 (1918), p. 65-73 et D.R. Theocharis, Prakt., 87 (1958), p. 64-80.

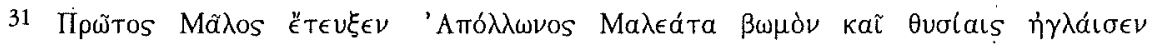
TÉ $\mu \epsilon \operatorname{vos}(1.27-28)$.

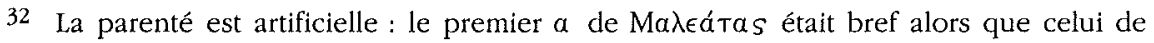

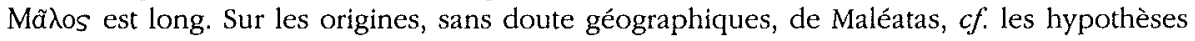
de M. Guarducci, I predecessori di Asclepio, in SMSR, 8 (1932), p. 215-229. 
daure, et notamment celui de Tricca. De sorte que la règle qui impose, à Tricca, un sacrifice sur l'autel d'Apollon Maléatas préalable à l'accomplissement du rite asclépieien de l'incubation apparaît comme un héritage épidaurien. Implicitement, le sanctuaire d'Asklépios à Tricca devient une filiale de celui d'Épidaure dont la primauté est ainsi affirmée.

La généalogie présentée par Isyllos a également pour but d'établir l'origine épidaurienne d'Asklépios, par sa mère. L'arrière-grand-père, Malos est, nous l'avons vu, le fondateur du sanctuaire d'Apollon Maléatas. Le grand-père est Phlégyas qui « habitait Épidaure, sa patrie ». Cette simple juxtaposition s'oppose en fait aux différentes traditions sur l'origine de Phlégyas : ce personnage, père de Coronis dans les différentes versions de la généalogie d'Asklépios, est le plus souvent considéré comme un Thessalien ${ }^{33}$. Une autre tradition entendue à Épidaure même par Pausanias fait de Phlégyas un étranger venu dans le Péloponnèse pour en espionner les habitants et y faire une razzia dans le but de l'envahir plus tard; au cours de cette expédition de reconnaissance, il était accompagné de sa fille Coronis, enceinte des œuvres d'Apollon, qui mit au monde, à Épidaure, l'enfant Asklépios qu'elle abandonna ${ }^{34}$. Donc, même dans cette version qui s'emploie à faire d'Épidaure le lieu de naissance d'Asklépios, Coronis demeure fille d'un étranger de passage.

Selon le péan d'Isyllos, en revanche, la fille de Phlégyas, la mère d'Asklépios, est une Épidaurienne. C'est ce dernier trait qui ressurgit dans l'adresse

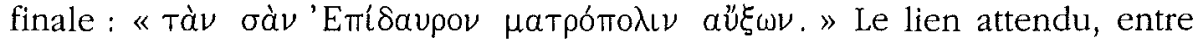
argument et pétition, se fait sur le mode suivant : puisque tu es épidaurien par ta mère, alors utilise tes pouvoirs pour protéger Épidaure. Dans la présentation généalogique, Isyllos précise que, si la mère d'Asklépios s'appelle Aigla, « à cause de sa beauté, elle était aussi appelée Coronis ${ }^{35}$. Ce nom d'Aigla

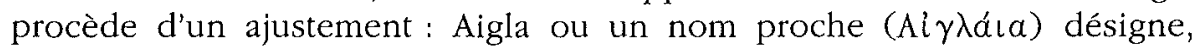
quoique de façon exceptionnelle, une fille d'Asklépios dans certaines tradi-

33 Dans la tradition transmise par PIND., Pyth., III, 1-61, il est roi des Lapithes, habitant à Lakéreia, près du lac Boibéis en Thessalie; $c f$. G. Solimano, Le aree del mito. Asclepio, Milan, 1976 , p. 25-36. On retrouve la même tradition dans l'Hymne bomérique à Asclépios et le péan gravé sur le monument de Sarapion à l'agora d'Athènes : IG II ${ }^{2}, 4510$; J.H. Oliver, The Sarapion monument and the paean of Sopbocles, in Hesperia 5 (1936), p. 112-114 et la remarque de S.B. Aleshire, The Atbenian Asklepieion, Amsterdam, 1989, p. 10; É. Des Places, La religion grecque. Dieux, cultes, rites et sentiment religieux dans la Grèce antique, Paris, 1969, p. 167-168.

34 PAus., II, 27, 3-4. La naissance d'Asklépios se conjugue alors avec le thème de l'enfant abandonné : celui-ci, exposé, est allaité par une chèvre et gardé par un chien. Le chien appartenait au berger Aresthanas; ce dernier, alors qu'il était à la recherche de ses bêtes, s'était enfui, ébloui et terrifié à la vue de l'éclair sorti du corps de l'enfant.

35 La justification du nom par la «beauté » est assez mystérieuse; korônis renvoie au sens propre à « ce qui est recourbé », « ligne recourbée », ce qui donne parfois le sens de « guirlande » ou « couronne ». 
tions $^{36}$. Al̈ $\gamma \lambda a$ est la forme dorienne de al $\gamma \lambda \eta$, qui représente l'éclat de la lumière, elle est « celle qui rayonne ». L'ajout d'un deuxième nom, celui de Coronis permet, quant à lui, de faire écho aux différentes traditions se rapportant à Asklépios où Coronis est la mère du dieu.

Mais Isyllos ajoute encore qu'Aigla est séduite par Apollon alors qu'elle se trouve dans la maison de Malos, son grand-père. Le choix du lieu, qui se justifie par les liens établis par le poète entre Malos et Apollon, permet d'établir qu'Asklépios, fils d'une Épidaurienne, a été conçu à Épidaure, ce qui ne peut que conforter la thèse de l'origine et de la naissance épidauriennes du dieu. Isyllos décrit ensuite les circonstances de cette naissance qu'il présente sur

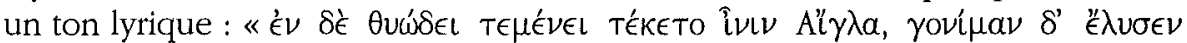

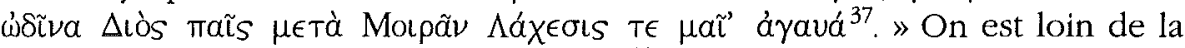
tonalité tragique du récit que fait Pindare ${ }^{38}$ de cette même naissance; Pindare reprend, on le sait, des traditions thessaliennes et béotiennes selon lesquelles Coronis, aimée d'Apollon et enceinte de ses œuvres, est séduite par un étranger, Ischys, fils d'Élatos. Apollon envoie alors Artémis pour châtier l'infidèle Coronis. Coronis est portée au bûcher mais Apollon détache l'enfant du cadavre de sa mère; il s'agit d'Asklépios, confié ensuite par son père au centaure Chiron qui lui apprend la médecine. Pindare fait ainsi de Coronis le symbole de ceux qui dédaignent le bonheur à leur portée pour démontrer qu'il faut se contenter de son sort surtout quand il est un don privilégié des dieux ${ }^{39}$. Isyllos rejette cette vision dramatique et fait d'Aigla-Coronis l'une de ces nombreuses mortelles séduites par Apollon. Elle accouche dans le téménos d'Apollon, ce qui implique que son accouchement n'est porteur d'aucune souillure. Elle est honorée par Apollon qui donne à son fils le nom

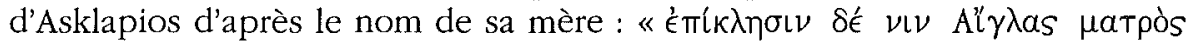

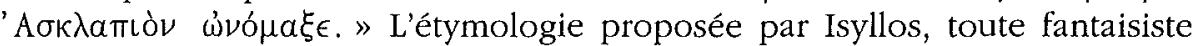
qu'elle soit, renforce encore les liens d'Asklépios à sa mère ${ }^{40}$.

Donc, au prix d'ajustements successifs des autres traditions véhiculées sur Asklépios, Isyllos parvient à faire de ce dernier le fils d'une Épidaurienne, n. 69 .

36 Ainsi dans le péan d'Érythrées. Cf. aussi U. von Wilamowitz, op. cit. (n. 3), p. 90,

37 1. 53-56. On trouve ici les Moires dans leurs fonctions de protectrices de la naissance, dans cet aspect qui les associe souvent à Ilithye, Artémis ou Héra. Sur la complexité de ces figures, $c f$. J. Villemonteix, Puissances de mort et de fécondité. À propos de la généalogie des Moires et des Heures, in Mort et fécondité dans les mythologies, Paris, 1986, p. 83-91. Ici, on notera que Lachésis est mise en avant, en étant séparée des Moires dont elle fait habituellement partie intégrante.

38 PIND., Pyth., III, 24-44.

39 St. Anastase, Apollon dans Pindare, thèse $3 e$ cycle de l'Université de ParisSorbonne, Athènes, 1975, p. 61-62.

40 L'étymologie d'Asklépios est inconnue et de nombreuses étymologies populaires ou savantes ont cherché à combler ce manque : cf. P. Chantraine, Dictionnaire étymolo-

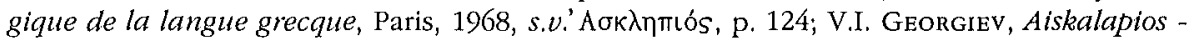
Asklépios, in AAntHung, 25 (1977), p. 317-319. 
conçu et mis au monde à Épidaure. Ce faisant, il adopte une position que l'on pourrait dire radicale dans les querelles qui ont vu le jour autour de l'origine d'Asclépios et qui ont opposé les Épidauriens tant aux Thessaliens qu'aux Messéniens et aux Arcadiens dans le courant du Iv ${ }^{\mathrm{e}}$ siècle. L'appui de l'oracle de Delphes dont il est fait expressément mention dans le prologue du péan ne surprend pas ${ }^{41}$, d'autant que Pausanias mentionne lui aussi une intervention de l'oracle destinée à légitimer l'origine proprement épidaurienne de la lignée d'Asklépios. Reprenant différentes versions de l'origine du dieu, Pausanias écrit en effet : «la troisième version est, à mon avis, la plus éloignée de la vérité; elle fait d'Asklépios le fils d'Arsinoé, la fille de Leukippos. Car, quand Apollophanés, l'Arcadien, vint à Delphes et demanda au dieu si Asklépios était le fils d'Arsinoé et partant, un Messénien, la Pythie donna cette réponse: "Ô Asklépios, né pour apporter un grand bonheur aux mortels, / Gage de l'amour mutuel dont j'ai joui avec la fille de Phlégyas, / Belle Coronis, qui te donna le jour dans la contrée sauvage, Épidaure." Cet oracle rendit tout à fait certain le fait qu'Asklépios n'était pas le fils d'Arsinoé et que l'histoire était une fiction inventée par Hésiode ou par un des scholiastes d'Hésiode, juste pour plaire aux Messéniens ${ }^{42}$. » D'après Pausanias, l'oracle a ainsi rejeté les prétentions de la Messénie - contestées par les Arcadiens - à faire d'Asklépios le fils d'Arsinoé, fille de Leukippos mais, surtout, il a répondu que c'est Coronis, fille de Phlégyas, qui a donné naissance à Asklépios à Épidaure. Dans cette réponse, l'origine de Phlégyas et de Coronis n'est pas précisée mais Épidaure est mentionnée explicitement comme lieu de naissance d'Asklépios. Cette consultation a probablement eu lieu peu de temps après la restauration de Messène, c'est-à-dire au moment où les Arcadiens éprouvèrent le besoin de contredire les prétentions des Messéniens dont les fresques peintes à l'arrière du temple de Messéné faisaient entrer Asklépios dans la généalogie des rois de Messénie ${ }^{43}$. C'est donc pour renforcer la position d'Épidaure dans cette polémique qu'Isyllos fait

41 L. 32-36 avec une formule qui appartient peut-être au style officiel de l'oracle : $c f$. P. Amandry, La mantique apollinienne à Delpbes. Essai sur le fonctionnement de l'oracle, Paris, 1950 (BEFAR 170), p. 154 et H.W. PARKE - D.E.W. Wormell, The Delpbic Oracle, Oxford, 1956, I, The History, p. 144; II, The Oracular Responses, $\mathrm{n}^{\circ} 279$. Pour les autres interventions de l'oracle de Delphes en faveur du culte d'Asklépios à Épidaure, $c f$. A. Burford, The Greek Temple Builders in Epidaums, Liverpool, 1969, p. 18 pour qui l'oracle aurait manifesté son approbation à la construction du temple du ive siècle. L'oracle intervient aussi dans la fondation du culte à Halieis dans le dernier quart du $\mathbf{I V}^{\mathrm{e}}$ siècle et peut-être même à Rome en 293 av. J.-C. : H.W. PARKE - D.E.W. Wormell, op. cit., I, p. 274,345 et II, no 277 et 353 .

42 Paus., II, 26, 7; H.W. Parke - D.E.W. Wormell, op. cit. (n. 41), I, p. 344-345 et II, $\mathrm{n}^{\circ}$ 276. Sur la version messénienne de la généalogie d'Asklépios, nous renvoyons à notre étude : A propos de l'Asclépieion de Messène: Asclépios poliade et guérisseur, in REG 110 (1997), p. 1-24.

43 Paus., IV, 31, 12. 
graver son péan ${ }^{44}$ mais la version qu'il tente ainsi d'accréditer va plus loin puisque, si elle fait naître Asklépios à Épidaure, elle l'intègre en outre à une famille épidaurienne.

Il reste que, par ce péan, Isyllos dispose aussi d'un support et d'une forme qui lui permettent d'exalter un certain nombre de valeurs conformes cette fois à l'idéal aristocratique qu'il défend. La généalogie du péan précise d'abord que Phlégyas a pris pour épouse Kléophéma, fille d'Ératô et de Malos; or, ce nom apparaît comme un composé où résonnent les termes de " gloire », kléos, et de «bruit », de « rumeur », phêmê ${ }^{45}$, dont elle est indissociable. On sait que le kléos est une des notions qui entrent dans la définition de l'agatbos: c'est au sens propre « ce qui est entendu », la renommée, le bouche-à-oreille qui se propage et, en particulier, la renommée qui récompense l'exploit, notamment guerrier. C'est en définitive la « gloire » telle qu'elle court de bouche en bouche, de génération en génération pour monter jusqu'aux dieux ${ }^{46}$. Dans ce contexte, la mention d'Aigla comme mère d'Asklépios ne surprend pas non plus : aigla, celle qui rayonne, n'est pas sans évoquer le kudos, l'éclat extérieur, visible, dont est illuminé le guerrier aristocratique qui a remporté la victoire. Kudos et kléos sont deux valeurs essentielles et complémentaires qui signalent le guerrier aristocratique ${ }^{47}$. Une telle utilisation de ces notions propres à l'idéologie aristocratique rattache ainsi Asklépios lui-même au projet politique que le poète défend dans l'ensemble de l'inscription, à savoir la promotion des valeurs et des institutions aristocratiques au sein de la cité. Ainsi, pour la procession qu'Isyllos a luimême instituée en l'honneur d'Apollon et d'Asklépios, la cité doit choisir, dans chaque tribu, les citoyens les meilleurs, par leur arétè (valeur, et dans son sens traditionnel, valeur qui repose sur la force guerrière) et leur aidôs (le respect comme mélange de modération et de sagesse $)^{48}$. La prière que les hommes d'Épidaure doivent prononcer à Apollon et à Asklépios demande aux divinités, non seulement la santé, mais «que la kalokagatbia des hommes d'Épidaure l'emporte toujours, ainsi que l'eunomia, la paix et une richesse irréprochable ${ }^{49}$. Dans le récit qui suit le péan, le poète affirme avoir vu, alors qu'il était enfant, Asklépios accourir d'Épidaure pour sauver Sparte attaquée par Philippe, attribuant.ainsi à la divinité le salut de cette cité. Pour

44 Il est bien difficile d'affirmer que ce péan était ainsi de nature à mettre un terme à la polémique: G. Solimano, op. cit. (n. 33), p. 83.

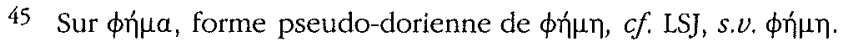

46 A. Fouchard, Aristocratie et démocratie. Idéologies et sociétés en Grèce ancienne, Besançon, 1997, p. 139. Il n'est donc pas surprenant qu'Isyllos ait fait de la mère de Kléophéma une Muse.

47 M. Detienne, les maîtres de vérité, Paris, 1981, p. 19-20; A. Fouchard, op. cit. (n. 46), p. 140 : «Si le kléos en met plein les oreilles, le kudos en met plein la vue».

48 L. $10-21$.

49 L. $23-24$. 
Isyllos, Asklépios intervient d'abord parce qu'il soutient les rois de Sparte, « honorant la race d'Héraclès que Zeus épargnait donc ainsi ». Puis parce que les Lacédémoniens " gardent de façon juste les oracles de Phoibos que Lycurgue, après avoir consulté l'oracle, imposa à la cité ». C'est dans cette mesure qu'à Sparte, Asklépios participe, à la hauteur de son arétè, à la résistance qu'oppose la cité à Philippe. L'arétè d'Asklépios peut s'entendre au sens de puissance ou de pouvoir ${ }^{50}$. Cependant, dans le contexte, le terme rappelle aussi la valeur qui, jusqu'à l'époque classique, repose d'abord, dans l'idéologie aristocratique, sur la force guerrière dont Asklépios précisément a fait preuve ici.

L'inscription dans son ensemble apparaît donc comme une entreprise d'exaltation d'un idéal aristocratique et trouve son aboutissement logique dans la louange de la constitution lacédémonienne et la défense de la politique antimacédonienne de Sparte. Dans ce projet, le péan devient une prière destinée à faire d'Asklépios un garant de cet idéal politique pour la cité d'Épidaure où le poète lui-même occupe une fonction bien précise. Car il est remarquable qu'avant de dérouler le récit de la généalogie d'Asklépios, Isyllos prend soin d'avertir qu'il n'en est pas l'auteur : « voici ce que dit l'histoire ( $\dot{\eta}$ $\phi a ́ \tau ı s)$ qui vint aux oreilles de nos ancêtres. " Il transforme ainsi ce récit généalogique en un thème digne d'être entendu, assimilable à un morceau d'épopée que lui, poète, ne fait que transmettre. Mais ce faisant, il se place dans la tradition de l'aède dont la fonction est précisément de transmettre le kléos des héros (dans la tradition de l'lliade et de l'Odyssée) ou celui des dieux bienheureux (dans la tradition d'une Théogonie) ${ }^{51}$. Or, si l'aède écoute et entend les Muses lui réciter le kléos, c'est bien lui qui chante devant l'auditoire. De la même façon, si Isyllos a su capter la rumeur qui lui enseigne la généalogie d'Asklépios, c'est bien lui qui fait inscrire le péan sur la pierre. Une façon d'être, à son tour, "maître du kléos ».

Pierre SINEUX

Université de Caen

Esplanade de la Paix

F - 14032 CaEn Cedex

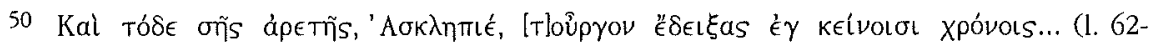

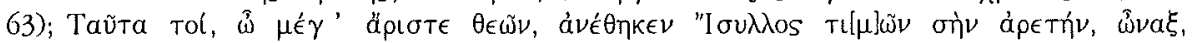

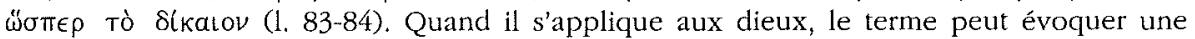
notion assez générale d'action divine, notion qui connote une idée de puissance; c'est sur ce sens-là que se fonde l'expression arétalogie qui apparaît comme la louange de l'ensemble des arétai, des pouvoirs bienfaisants d'une divinité; cf. Y. Grandjean, Une nouvelle arétalogie d'Isis à Maronée, Leiden, 1975, p. 1-8.

51 G. NAGY, Le mellleur des Acbéens, trad. française, Paris, 1994, p. 130. 